\title{
Produção e qualidade de frutos de mirtileiros sob diferentes intensidades de poda
}

\author{
Production and fruit quality of blueberries under different pruning intensities
}

\author{
André Luiz Külkamp de Souza ${ }^{I}$ Robson Rodrigues PereiraII Samila Silva Camargo ${ }^{\text {II }}$ \\ Doralice Lobato de Oliveira Fisher ${ }^{\text {III }}$ Márcia Wulff Schuch ${ }^{\text {II }}$ Mateus da Silveira Pasa ${ }^{\mathrm{IV}}$ \\ Juliano Dutra SchmitzII
}

RESUMO

O sistema de poda do mirtileiro (Vaccinium ashei Read) utilizado no país se fundamenta em estudos realizados em locais com condições edafoclimáticas distintas da nossa realidade. Assim, foram avaliadas diferentes intensidades de poda de frutificação em dois genótipos de mirtileiro, e seus efeitos na produção e na qualidade dos frutos. O experimento foi conduzido em pomar comercial, localizado em Pelotas- $R S$. O delineamento experimental foi o de blocos casualizados completos, em esquema fatorial (2x4), com quatro repetições. Plantas de sete anos de idade, do grupo Rabbiteye, cultivares 'Briteblue' e 'Woodard', foram submetidas às intensidades de poda leve, moderada, drástica e sem poda (testemunha) nos anos de 2010 e 2011, no período de repouso hibernal (primeira quinzena de agosto). Foram avaliadas a produção média por planta, sendo estimada a produtividade por área, peso fresco, diâmetro, teor de sólidos solúveis, $\mathrm{pH}$ $e$ acidez titulável dos frutos. De acordo com os resultados, a intensidade de poda tem efeito sobre a antecipação da colheita. Com a decorrência de uma maior intensidade de poda, houve uma tendência de aumento do tamanho dos frutos. Para mirtileiros Briteblue, há decréscimo de produtividade, conforme se aumenta a intensidade de poda. Mirtileiros Briteblue são mais produtivos e apresentam maior matéria fresca de fruto do que Woodard.

Palavras-chave: Vaccinium ashei, mirtilo, poda de inverno.

\section{ABSTRACT}

The blueberry (Vaccinium ashei Read) pruning system currently used is based on studies performed at edaphoclimatic conditions different of our reality. Thus, it was assessed the effects of different fructification pruning intensities on production and fruit quality of two blueberry genotypes. The trial was performed in a commercial orchard in Pelotas - RS. The experimental design was a complete randomized block, in a factorial design (2x4) with four replications. Seven year old plant of Rabbiteye group, cultivars 'Briteblue' e 'Woodard', were undergone to the pruning intensities light, moderate drastic and no pruning (control) during the winter of 2010 and 2011 (first two weeks of august). The parameters assessed were average plant yield, estimated yield per area, fruit fresh weight, fruit diameter, soluble solids, $\mathrm{pH}$ and titratable acidity. According to the results, pruning intensity affects harvest anticipation. As the pruning intensity increased, there was a trend of increased fruit size. The productivity of Briteblue blueberries decreases as pruning intensity increases. Briteblue are more productive and show greater fresh fruit weight than Woodard blueberries.

Key words: Vaccinium ashei, blueberry, winter prunning.

\section{INTRODUÇÃO}

Apreciado pelo seu sabor exótico, propriedades nutricionais e medicinais, o mirtilo (Vaccinium ashei) tem se destacado nos últimos anos por ser um fruto rico em antocianinas (CONNOR et al., 2002), componente que auxilia no combate aos radicais livres. Apresenta alta rentabilidade, porém um dos entraves na produção é a grande demanda de mão-de-obra, principalmente na poda e colheita, encarecendo o seu custo (NÚÑEZ, 2009).

No Brasil, o cultivo do mirtileiro é recente, necessitando de estudos específicos de manejo, nas

\footnotetext{
IEmpresa de Pesquisa Agropecuária e Extensão Rural de Santa Catarina, Estação Experimental de Videira, Rua João Zardo 1660, Bairro Campo Experimental, 89560-000, Videira, SC, Brasil. E-mail: andreluizks@ yahoo.com.br. Autor para correspondência.

IIPrograma de Pós-graduação em Agronomia, Departamento de Fitotecnia, Universidade Federal de Pelotas (UFPel), Pelotas, RS, Brasil.

III Instituto Federal Sul-Rio-Grandense (IFSul), Pelotas, RS, Brasil.

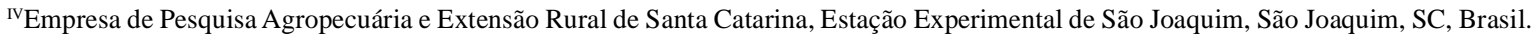
Recebido 18.02.13 Aprovado 19.05.14 Devolvido pelo autor 10.07.14 CR-2013-0211.R2
} 
diferentes condições edafoclimáticas, para os diversos grupos (Rabbiteye, Southern Highbush e Northern Highbush). Dentre estes estudos, a consolidação do sistema adequado de poda é fundamental para o crescimento da cultura no país.

A poda de frutificação é realizada no período de repouso da planta e serve para diminuir a alternância de produção, prevenindo a frutificação excessiva. Desse modo, mantêm-se o padrão em tamanho e qualidade dos frutos (LOCKWOOD, 1999; WILLIAMSON et al., 2004). A intensidade da poda de frutificação determina como será o crescimento vegetativo durante a primavera e verão e, quanto mais drástica, maior número de ramos vegetativos e menor quantidade de frutos serão formados (ALBERT et al., 2010).

Devido à escassez de estudos referentes ao manejo de poda de mirtileiro específico para as condições do Sul do Brasil, o setor produtivo utiliza informações não validadas pela pesquisa nacional. Dessa forma, com intuito de estabelecer um critério de poda adequado para plantas do grupo Rabbiteye, nas condições do Rio Grande do Sul, foi avaliado o efeito de diferentes intensidades de poda de frutificação sobre a produtividade e a qualidade dos frutos de mirtileiros 'Briteblue' e 'Woodard'.

\section{MATERIAL E MÉTODOS}

O experimento foi realizado nos anos de 2010 e 2011, em pomar comercial, localizado no município de Pelotas-RS (31 33 'S, 52²3'O; $110 \mathrm{~m}$ de altitude), em plantas adultas dos genótipos Briteblue e Woodard, pertencentes ao grupo Rabbiteye. O solo do campo experimental é Argisolo vermelho-amarelo e, segundo classificação de Köppen, a região de Pelotas apresenta clima do tipo Cfa, (C) clima temperado quente, (f) de chuvas bem distribuídas e (a) verão suave.

O pomar foi plantado no ano de 2003, em espaçamento de $1,5 \mathrm{~m}$ entre plantas e $4 \mathrm{~m}$ entre linhas, totalizando 1.667 plantas ha- ${ }^{-1}$. A adubação foi realizada igualitariamente para todos os tratamentos, conforme as exigências da cultura. $\mathrm{O}$ manejo das plantas daninhas foi realizado através de roçada nas entre linhas de plantio, enquanto que nas linhas foram realizadas capinas manuais. Quando houve estiagem, as plantas foram irrigadas através de gotejamento para evitar restrições hídricas ao cultivo. As precipitações nos anos de 2010 e 2011 foram de 1.325 e $1.000 \mathrm{~mm}$, respectivamente.

No final da primeira quinzena de julho, durante o período de repouso das plantas, foi realizada aplicação de calda sulfocálcica a $10 \%$ de concentração, sendo desnecessária a aplicação de qualquer outro defensivo agrícola. Durante os anos anteriores ao experimento, foram realizadas, pelo proprietário, podas de inverno e verão, visando à formação e o equilíbrio da vegetação/produção da planta, sendo a poda verde realizada logo após a colheita.

$\mathrm{O}$ experimento foi realizado em delineamento de blocos casualizados completos, em arranjo fatorial $2 \times 4$, com quatro repetições. Plantas dos genótipos Briteblue e Woodard foram submetidas a quatro intensidades de poda: drástica, moderada, leve e sem poda.

As podas foram praticadas em dois anos consecutivos, 2010 e 2011, sempre na primeira quinzena de agosto. No tratamento testemunha, não foi realizada a poda de frutificação, permanecendo todos os ramos, inclusive os secos que produziram no último ciclo de produção. A Poda leve consistiu na retirada de aproximadamente $25 \%$ dos ramos da planta, incluindo os que produziram na última safra, mal posicionados, secos e extremamente finos. A Poda moderada consistiu na retirada de cerca de $50 \%$ dos ramos, deixando apenas aqueles com maior potencial produtivo (espessura acima de $8 \mathrm{~mm}$ ); foram eliminados ramos sem gema floral e hastes mais velhas, para promover a sua renovação. A Poda drástica consistiu na realização de uma poda rigorosa, caracterizada pela retirada de aproximadamente $75 \%$ dos ramos, mantendo-se somente a estrutura principal da planta, ou seja, as hastes vindas de sua base; retiraram-se as mal localizadas no interior da planta e algumas foram podadas a $50 \mathrm{~cm}$ do solo para estimular brotações mais baixas no intuito de facilitar a colheita.

Aos 112 dias após a poda, iniciou-se a colheita dos frutos no estádio de maturação completo, com coloração violeta (em todo o fruto, inclusive na inserção do pedúnculo) e presença de pruína. As colheitas foram realizadas semanalmente e os frutos acondicionados em bandejas de plástico e, em seguida, levados ao Laboratório de Fruticultura do Departamento de Fitotecnia, da Universidade Federal de Pelotas. Foram avaliados o número de frutos colhidos por planta, a matéria fresca dos frutos (MFF), o diâmetro médio dos frutos (DMF), o teor de sólidos solúveis (SS), o pH e a acidez titulável (AT). Também foi estimada a produção média por planta, a produtividade por área e produção de frutos por data de colheita.

A produção média por planta $(\mathrm{kg})$ foi realizada através da pesagem dos frutos colhidos por parcela, divididos pelo número de plantas (duas). Baseada na densidade de plantio de 1.667 plantas $\mathrm{ha}^{-1}$, foi estimada a produtividade ( $\left.\mathrm{t} \mathrm{ha}{ }^{-1}\right)$. A MFF foi avaliada em balança de precisão, através da pesagem 
(g) de todos os frutos colhidos. Após a pesagem e contagem, foi obtida, de maneira aleatória, uma amostra de 100 frutos por repetição, para a obtenção do DMF (mm), SS (\%), pH e AT (meq $\left.100 \mathrm{~mL}^{-1}\right)$. O DMF foi medido no seu sentido equatorial, através do uso de paquímetro digital. Os teores de SS foram determinados através de refratômetro digital e os dados expressos em porcentagem $(\%)$. Adeterminação do $\mathrm{pH}$ foi realizada em pHmetro digital e a AT por titulometria, sendo utilizados $10 \mathrm{~mL}$ de suco, diluídos em $90 \mathrm{~mL}$ de água destilada e titulados com hidróxido de sódio a $0,1 \mathrm{~N}$ até $\mathrm{pH} 8,1$.

Os dados obtidos foram submetidos ao teste de normalidade, Shapiro-Wilk. Realizouse análise de variância e, quando significativas, as médias dos tratamentos foram comparadas pelo teste de Tukey, em nível de $5 \%$ de probabilidade.

\section{RESULTADOS E DISCUSSÃO}

Nos dois anos de avaliação, constatou-se que o mirtileiro 'Briteblue' apresentou maior produção por planta e produtividade por área, com exceção da poda drástica na primeira safra (Tabela 1). Com relação ao tamanho dos frutos (diâmetro), foi constatada diferença entre os genótipos apenas para os mirtileiros não podados na segunda safra. A cultivar 'Woodard' apresentou menor matéria fresca dos frutos nos dois anos de estudo. A maior produção de Briteblue se deve, provavelmente, as suas características genéticas e de adaptabilidade, fato confirmado por ANTUNES et al. (2008) em estudos com as mesmas cultivares na região de Pelotas-RS. O mirtileiro Woodard apresentou menor pH na safra 2010/2011 e maior teor de AT nas duas safras (Tabela 2), atributos que tornam essa cultivar interessante para industrialização, já que os ácidos orgânicos influenciam no sabor, odor, estabilidade e na manutenção de qualidade (RODRIGUES et al., 2007).

Nos dois anos de estudo, a menor matéria fresca dos frutos de 'Briteblue' foi observada na testemunha, enquanto que, para 'Woodard', a testemunha foi inferior apenas à poda drástica (Tabela 1). Nas plantas em que não foi realizada poda (testemunha), notou-se menor diâmetro dos frutos na safra 2011 para Woodard. De acordo com SPIERS et al. (2002) e NIEUWENHUIS (1993), a ausência de poda resulta em frutos pequenos e alta produção, além de baixo crescimento dos novos ramos, que serão responsáveis pela produção no ano seguinte. Além disso, a grande quantidade de ramos no interior da planta e a presença de ramos excessivamente altos e baixos dificultaram a colheita e comprometeram a arquitetura das plantas. STRIK et al. (2003), ao testar diferentes intensidades de poda, constataram que a eficiência da colheita de plantas não podadas foi reduzida em até $51 \%$, sendo que esta atividade é responsável pelo maior custo em mão de obra no cultivo de mirtilo.

Tabela 1 - Produção média por planta, produtividade estimada por hectare, matéria fresca e diâmetro dos frutos de mirtileiros 'Briteblue' (BT) e 'Woodard' (WD), submetidos a diferentes intensidades de poda de frutificação. Pelotas - RS, 2013.

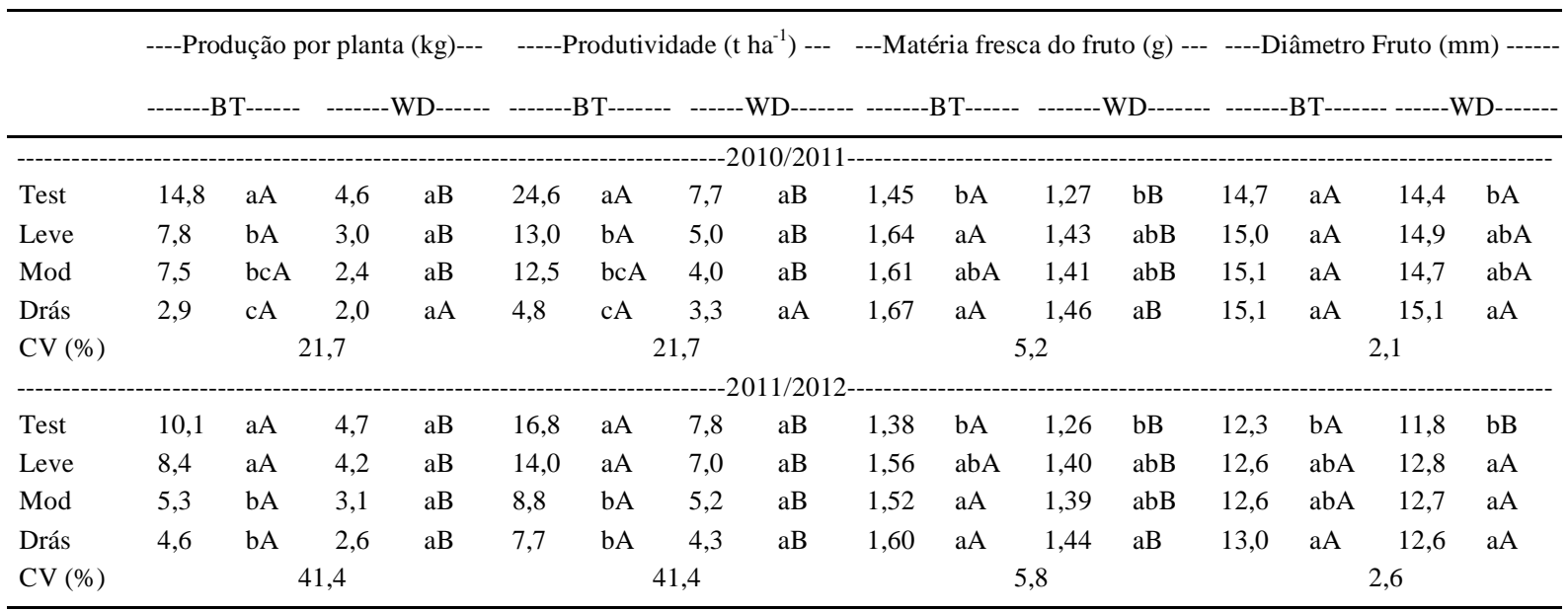

${ }^{1}$ Médias seguidas pela mesma letra minúscula na coluna e maiúscula na linha, não diferem pelo teste de Tukey com nível de significância de 5\%. Test: testemunha; Mod: moderada; Drás: drástica. 
Tabela 2 - Acidez titulável, pH e sólidos solúveis de frutos de mirtileiros 'Briteblue' (BT) e 'Woodard' (WD), submetidos a diferentes intensidades de poda de frutificação. Pelotas - RS, 2013.

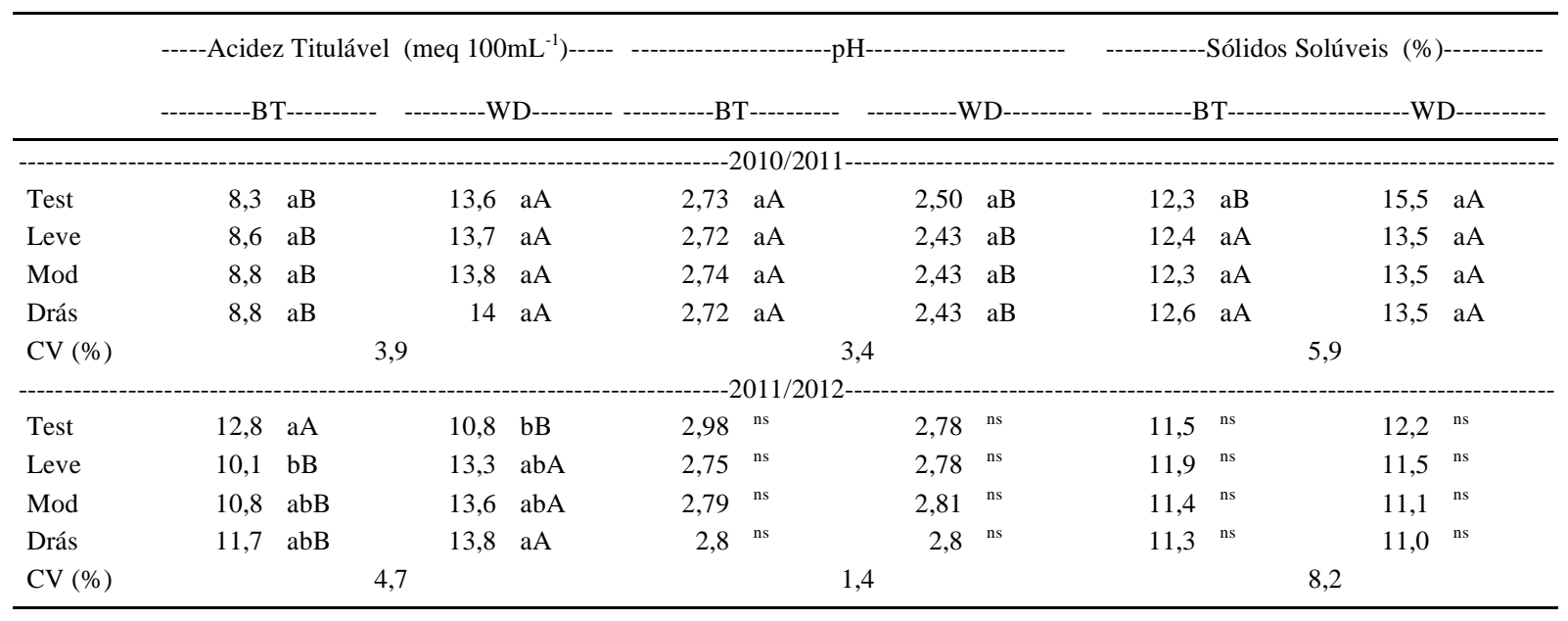

${ }^{1}$ Médias seguidas pela mesma letra minúscula na coluna e maiúscula na linha não diferem pelo teste de Tukey com nível de significância de $5 \%$. *ns: não significativo a $5 \%$ de probabilidade.

Test: testemunha; Mod: moderada; Drás: drástica

À medida que se realiza a poda de frutificação, há eliminação de ramos e consequentemente gemas florais, deixando menor quantidade de frutos e favorecendo que esses alcancem maior tamanho (DAVIES, 1983). De acordo com BAÑADOS (2005), a poda drástica resulta em crescimento vegetativo excessivo e poucos frutos, causando desbalanço entre a porção vegetativa e produtiva da planta. $\mathrm{O}$ maior crescimento vegetativo proporciona o surgimento de ramos mais vigorosos, capazes de produzir frutos maiores (JANSEN, 1997). As plantas podadas drasticamente produziram frutos mais agrupados e localizados nas extremidades dos ramos, facilitando a colheita. Esta característica se deve à menor quantidade de gemas florais por planta, permitindo que estas produzam maior quantidade de flores por inflorescência.

Para o mirtileiro 'Woodard', não houve diferenças significativas entre os tratamentos, nos dois anos de experimento, tanto para a produção como para a produtividade. Em 'Briteblue', a produção média por planta e produtividade estimada por hectare tenderam à queda, conforme aumentava a intensidade de poda (Tabela 1). A maior produção obtida nas plantas não podadas no primeiro ano não se repetiu no segundo, quando as podadas de forma leve obtiveram produções iguais estatisticamente. Segundo STRIK et al. (2003), a não realização da poda pode ser uma opção em curto prazo, mas tem efeitos indesejáveis em longo prazo, como a falta de renovação de ramos e frutos de baixa qualidade. Para 'Briteblue', as plantas submetidas à poda drástica apresentaram produção abaixo dos demais tratamentos, demonstrando não ser uma opção viável, a não ser em plantas fracas, com pouca brotação, nas quais esta modalidade pode ser realizada para rejuvenescer a planta, proporcionando o surgimento de ramificações novas. CORMACK \& WAISTER (1977), em estudos com mirtileiros do grupo Highbush (Vaccinium corymbosun), afirmaram que a poda drástica, realizada consecutivamente por três anos, não proporcionou maior produção e tamanho dos frutos, mesmo estas apresentando maior estrutura e vigor das novas brotações.

Analisando-se os períodos de colheitas (Figura 1), nota-se uma maior precocidade de produção no mirtileiro Woodard, em que, de modo geral, a colheita tem início e término com uma semana de antecedência em relação à Briteblue, não havendo coincidência no pico de produção, fator interessante para escalonar a colheita e melhorar o aproveitamento da mão-de-obra. Nas plantas de Woodard, independente dos tratamentos, o pico de colheita se localizou em meados do mês de dezembro, sendo que posteriormente a quantidade de frutos diminui até o seu fim na primeira quinzena de janeiro. Em contrapartida, na cultivar 'Briteblue', o pico de colheita é mais pronunciado e se encontra no fim de dezembro e início de janeiro. 


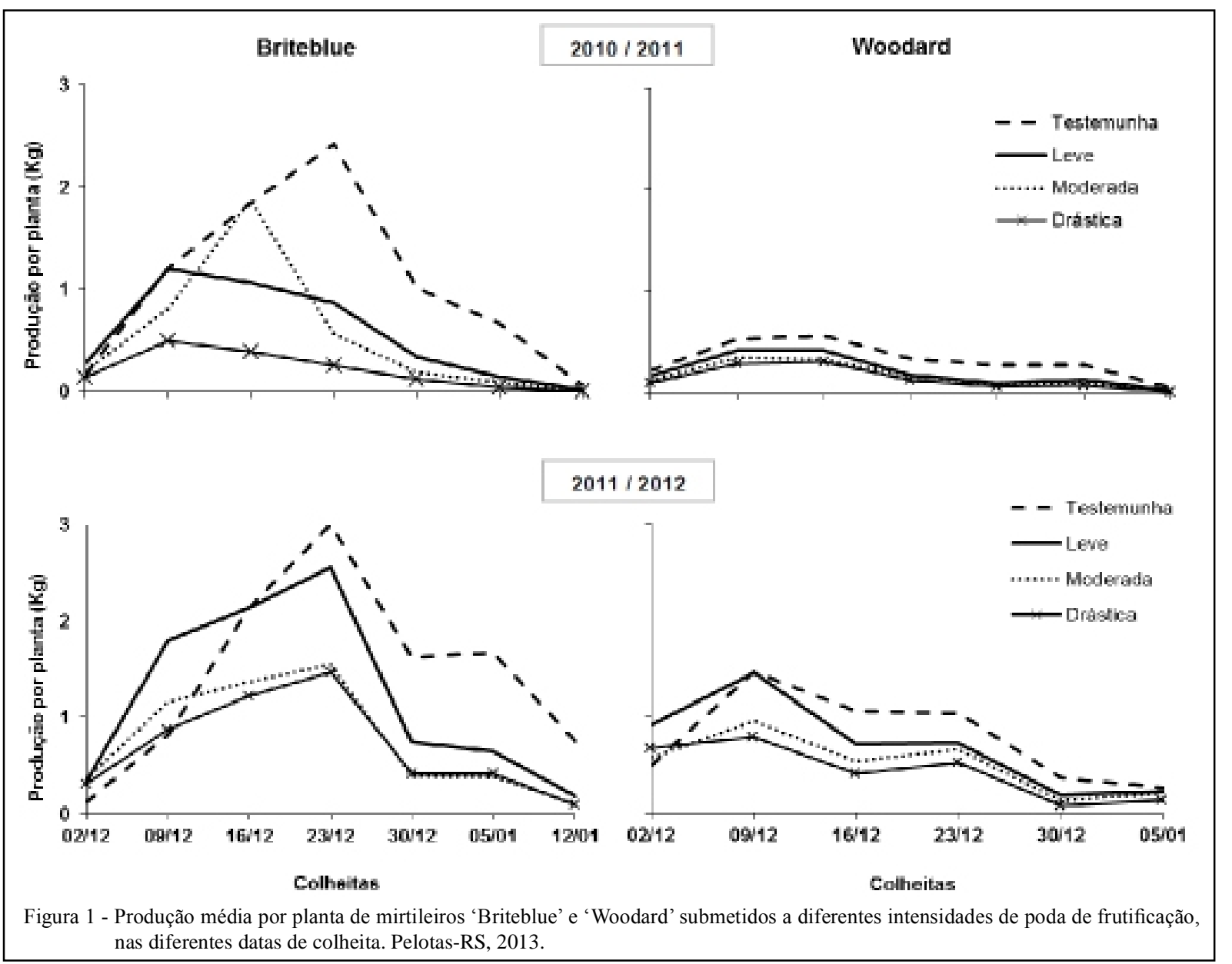

Afigura 2 mostra a distribuição da produção dos frutos de cada tratamento nas diferentes datas de colheita, quando as intensidades de poda interferiram quanto à distribuição da colheita no tempo. Notase que, nas duas primeiras datas de colheita, a porcentagem de frutos colhidos das plantas que não foram submetidas à poda (testemunha) foi inferior aos demais tratamentos. Em contrapartida, no terço final, esse tratamento se destacou, demonstrando que a intensidade de poda tem efeito sobre a antecipação da colheita. Esses resultados corroboram STRIK et al. (2003) e NIEUWENHUIS (1993) em estudos com plantas do grupo Highbush. De acordo com estes autores e com os resultados observados neste trabalho, plantas não podadas obtiveram menor porcentagem de produção nas primeiras colheitas, diferente do que ocorreu nas últimas colheitas, quando houve maior concentração da produção.

A prática de poda teve pouca influência sobre a qualidade dos frutos avaliados (Tabela 2), já que o pH e o teor de sólidos solúveis não foram alterados nas avaliações. A acidez titulável foi influenciada pela prática da poda, mas, apenas no ciclo 2011/2012, sendo variável, dependendo da cultivar. Ao contrário do que se imaginava, a intensidade de poda não influenciou o teor de sólidos solúveis, contrariando resultados encontrados por KUMAR et al. (2010) na cultura do pessegueiro, onde plantas podadas de forma rigorosa apresentaram maior teor de SS e AT, devido à maior insolação na planta e menor quantidade de frutos. Frutos de mirtileiros 'Woodard' apresentaram maior acidez titulável e menor pH na safra 2010/2011, quando comparado a 'Briteblue'.

A escolha da intensidade de poda pelo produtor deve priorizar os aspectos produtivos ou que facilite o manejo do pomar, levando-se em consideração o vigor das plantas e o destino da produção. O maior tamanho de frutos é atributo essencial para mercado in natura, seja ele interno ou externo. Já para o processamento industrial, a produtividade é o fator mais relevante, desde que os frutos tenham um tamanho aceitável e que compense o custo da mão-de-obra na colheita. 


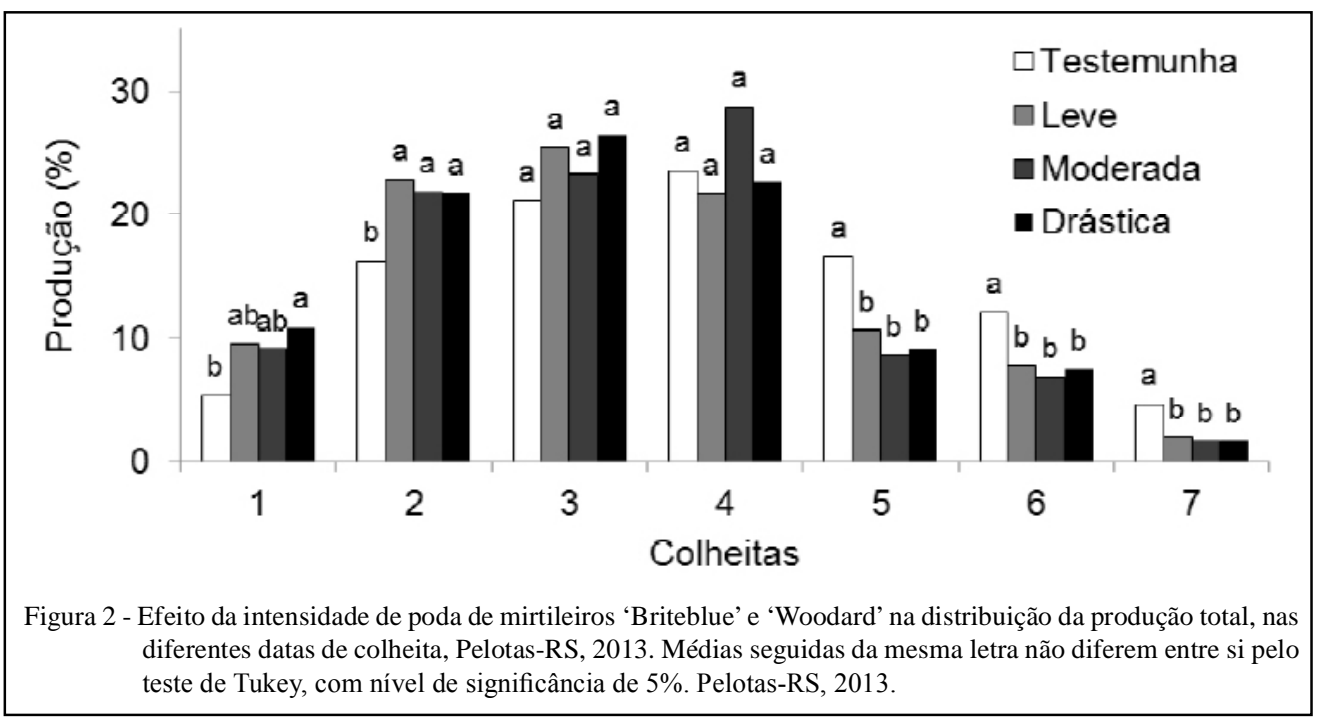

\section{CONCLUSÃO}

Para 'Briteblue', há decréscimo de produtividade conforme se aumenta a intensidade de poda. Mirtileiros dessa cultivar são mais produtivos e apresentam maior matéria fresca de fruto do que 'Woodard'. Além disso, para ambas as cultivares, a poda antecipa a colheita dos frutos.

Há tendência de aumento do tamanho dos frutos quanto maior a intensidade de poda, entretanto, essa não influencia o teor de sólidos solúveis dos frutos.

\section{AGRADECIMENTOS}

Ao Conselho Nacional de Desenvolvimento Científico e Tecnológico (CNPq) e à Coordenação de Aperfeiçoamento de Pessoal de Nível Superior (CAPES), pelas bolsas concedidas, e à empresa Frutplan, pela disponibilização do pomar e auxílio no estudo.

\section{REFERÊNCIAS}

ALBERT, T. et al. The effect of mulching and pruning on the vegetative growth and yield of the half-high blueberry. Agronomy Research, v.8, n.1, p.759-769, 2010. Disponível em: <http:// agronomy.emu.ee/vol081/p8101.pdf>. Acesso em: 30 maio, 2012.

ANTUNES, L.E.C. et al. Fenologia, produção e qualidade de frutos de mirtilo. Pesquisa Agropecuária Brasileira, v.43, n.8, p.1011-1015, 2008. Disponível em: <http://www.scielo.br/ pdf/pab/v43n8/v43n8a09.pdf>. Acesso em: 10 fev. 2012. doi: 10.1590/S0100-204X2008000800009.

BAÑADOS, P.O. Claves para la poda de arándanos. Revista Agronomía y Forestal UC, v.7, p.28-31, 2005. Disponível em: <http://agronomia.uc.cl/index.php〉. Acesso em: 01 nov. 2011.

CONNOR, A.M. et al. Changes in fruit antioxidant activity among blueberry cultivars during cold-temperature storage. Journal of Agricultural Food Chemistry, v.50, p.893-898, 2002. Disponível em: <http://pubs.acs.org/doi/abs/10.1021/jf011212y>. Acesso em: 02 fev. 2012. doi: 10.1021/jf011212y.

CORMACK, M.R.; WAISTER, P.D. Some effects of variety, pruning and $\mathrm{pH}$ adjustments on the early development of highbush blueberry plants in Scotland. Acta Horticulturae, v.61, p.6984, 1977. Disponível em: <http://www.pubhort.org/actahort/ books/61/61_8.htm>. Acesso em: 12 out. 2012.

DAVIES, F.S. Pruning, yield and morphology of 3 rabbiteye blueberry cultivars in Florida. Proceedings of the Florida State Horticultural Society, v.96, p.192-195, 1983. Disponível em: <http://www.fshs. org/Proceedings/Password\%20Protected/1983\%20Vol.\%2096/192195\%20(DAVIES).pdf>. Acesso em: 28 dez. 2011.

JANSEN, W.A.G.M. Pruning of highbush blueberries. Acta Horticulturae, v.446, p.333-335, 1997. Disponível em:<http:// www.actahort.org/books/446/446_49.htm>. Acesso em: 03 mar. 2012.

KUMAR, M. et al. Effect of pruning intensity on peach yield and fruit quality. Scientia Horticulturae, v.125, p.218-221, 2010. Disponível em: <http://www.sciencedirect.com/science/article/pii/ S0304423810001391>. Acesso em: 01 fev. 2012. doi: 10.1016/j. scienta.2010.03.027.

LOCKWOOD, D.W. Pruning blueberries. Agricultural Extension Service, The University of Tennessee, v.1, p.0104, 1999. Disponível em: <https://utextension.tennessee.edu/ publications/Documents/SP284-E.pdf>. Acesso em: 19 jan. 2012.

NIEUWENHUIS, J.C. Pruning systems in highbush blueberries. Acta Horticulturae, v.346, p.173-177, 1993. Disponível em: <http://www.actahort.org/books/346/346_23.htm>. Acesso em: 06 jan. 2012.

NÚÑEZ, E.E. Viabilidade econômica da produção de mirtilo. 2009. 39f. Monografia (Especialização em Agronegócio) - Escola Superior de Agricultura "Luiz de Queiroz", Universidade de São Paulo, SP.

RODRIGUES, S.A. et al. Influência da cultivar nas característica físicas, químicas e sensoriais de topping de mirtilo. Revista 
Brasileira de Tecnologia Agroindustrial, v.01, p.9-29, 2007. Disponível em: <http://revistas.utfpr.edu.br/pg/index.php/rbta/ article/view/245>. Acesso em: 13 dez. 2011. doi: 10.3895/S198136862007000100002 .

SPIERS, J.M. et al. Effects of pruning on 'Climax' Rabbiteye Blueberry. Acta Horticulturae, v.574, p.133-237, 2002. Disponível em: <http://www.actahort.org/books/574/574_35. htm>. Acesso em: 09 nov. 2011.
STRIK, B. et al. Pruning severity affects yield, berry weight, and hand harvest efficiency of highbush blueberry. Hortscience, v.38, n.2, p.196-199, 2003. Disponível em: <http://hortsci. ashspublications.org/content/38/2/196.full.pdf >. Acesso em: 07 jan. 2012.

WILLIAMSON, J.G. et al. Pruning blueberry plants in Florida, v.1, p.01-05, 2004. Disponível em: <http://edis.ifas.ufl.edu/ pdffiles/HS/HS22300.pdf>. Acesso em: 20 jan. 2012. 